\title{
miR-30c-5p inhibits glioma proliferation and invasion via targeting $\mathrm{Bcl} 2$
}

\author{
Li-Qun Yuan, Tan Zhang, Liang Xu, Hui Han, Shi-Hai Liu
}

Neurosurgery Department, The Second Affiliated Hospital of Soochow University, Suzhou, China

Contributions: (I) Conception and design: LQ Yuan, SH Liu; (II) Administrative support: L Xu, H Han; (III) Provision of study materials or patients: T Zhang, L Xu; (IV) Collection and assembly of data: LQ Yuan, H Han; (V) Data analysis and interpretation: LQ Yuan, T Zhang; (VI) Manuscript writing: All authors; (VII) Final approval of manuscript: All authors.

Correspondence to: Shi-Hai Liu. Neurosurgery Department, The Second Affiliated Hospital of Soochow University, Suzhou, China. Email: 986522387@qq.com.

Background: Glioma is a highly malignant brain tumor, characterized by the poor prognosis and high recurrence rates. Previous studies have confirmed that miRNA-30c-5p is closely associated with tumor cell biological properties. The present study explored the biological role of miR-30c-5p in human glioma malignant behavior and underlying mechanisms.

Methods: Levels of miR-30c-5p were detected in glioma tissues and adjacent normal tissues. Two glioma cell lines including U87 and U251 were transfected with miR-30c-5p mimic or inhibitors. Cell proliferation was evaluated by MTT assay and colony formation assay. Cell apoptosis and invasive potential of glioma cells were assessed by flow cytometry and transwell assays, respectively. Luciferase reporter assay was performed to validate the target gene of miR-30c-5p.

Results: Levels of miR-30c-5p were dramatically decreased in glioma tissues as compared to the adjacent normal tissues. Upregulation of miR-30c-5p significantly suppressed cell growth and colony formation, and induced apoptosis in glioma cells. In contrast, inhibition of miR-30c-5p promoted the proliferation and inhibited apoptosis in tumor cells. Furthermore, miR-30c-5p strongly suppresses the invasion of glioma cells. Western blot showed that Bcl-2 was significantly decreased following treatment with miR-30c-5p mimics and increased after miR-30c-5p inhibitor treatment. Moreover, luciferase reporter assays indicated that transfection of miR-30c-5p led to a marked reduction of luciferase activity, but had no effect on Bcl-2 3'-UTR mutated fragment. Mechanically, miR-30c-5p promoted the activation of caspase 3 and caspase 9 in glioma cells. Furthermore, miR-30c-5p promoted apoptosis and inhibited colony formation and migration, and knockdown of $\mathrm{Bcl} 2$ further increased the number of apoptotic cells and suppressed colony formation and migration of glioma cells. By contrast, miR-30c-5p inhibitors decreased apoptosis and increased colony formation and migration, and restored $\mathrm{Bcl} 2$ expression further suppressed glioma cell apoptosis and enhanced colony formation and migration.

Conclusions: These results demonstrated that miR-30c-5p regulated growth, apoptosis and migration in glioma cells by targeting Bcl2, suggesting that miR-30c-5p might serve as a novel target for glioma therapy.

Keywords: Glioma; miRNA; Bcl2

Submitted Dec 25, 2019. Accepted for publication Sep 17, 2020.

doi: $10.21037 /$ tcr-19-2957

View this article at: http://dx.doi.org/10.21037/tcr-19-2957 


\section{Introduction}

Glioma is the most common type of intracranial tumors characterized by invasive growth and aggressive metastasis capabilities (1). Based on the classification of the World Health Organization (WHO), glioma is divided into grade I to grade IV $(1,2)$. The brain glioma is considered as one of the most malignant tumors because of its resistance to chemotherapy and radiotherapy as well as the subsequent relapse. The detailed molecular mechanisms responsible for glioma initiation and progression are not fully elucidated, it is generally accepted that uncontrolled growth and migration of tumor cells contribute to glioma malignancy (3).

MicroRNAs (miRNAs), small non-coding RNAs (19-24 nucleotides in length), modulate downstream genes at the post-transcriptional level $(4,5)$. Specifically, miRNAs bind to the 3'-UTR of target mRNAs, leading to mRNA destabilization and/or translational inhibition $(6,7)$. Amounting studies confirmed that miRNAs are frequently deregulated in human malignancies and play diverse roles in tumor cell behaviors, including proliferation, migration, drug resistance, and epithelial mesenchymal transition (8). Functionally, miRNAs act as tumor suppressor genes or oncogenes to regulate the expression of tumor related genes, highlighting their potential values in cancer diagnosis and treatment.

MiR-30c, belonging to the miR-30 family, has been shown to be involved in a variety of tumors (9-11). In breast cancer, miR-30c serves as an independent predictor of endocrine therapy in advanced estrogen receptor (ER) positive cases $(12,13)$. Moreover, aberrant miR-30c expression changes the expressions of EMT markers and lead to the suppression of epithelial mesenchymal transition (EMT) (14). A recent study has confirmed that miR-30c-5p suppresses migration, invasion and EMT by targeting MTA1 in gastric cancer (15). In glioma cells, high expression levels of $\mathrm{miR}-21$ and $-30 \mathrm{~b} / \mathrm{c}$ are reported to maintain the tumor necrosis factor-related apoptosis-inducing ligand (TRAIL)resistant phenotype (16). The aforementioned studies suggest the critical role of miR-30c-5p in human cancers, including glioma. However, the effect of miR-30c on glioma progression and its underlying mechanism still needs further investigation. In the present study, we explored the role of miR-30c-5p in growth, apoptosis, migration of glioma cells, aiming to identify novel therapeutic target for treatment of glioma. We present the following article in accordance with the MDAR reporting checklist (available at http://dx.doi.org/10.21037/tcr-19-2957).

\section{Methods}

\section{Clinical specimens}

Twenty paired human glioma specimens and the adjacent normal tissues were obtained from Department of Neurosurgery, the Second Affiliated Hospital of Soochow University. All samples were obtained at the time of surgery and immediately stored in liquid nitrogen until use. The study was conducted in accordance with the Declaration of Helsinki (as revised in 2013). This study was approved by the Ethics Committee of the Second Affiliated Hospital of Soochow University [No. JD-LK-2018-027-02], and all participants provided written informed consent.

\section{Cell culture}

The glioma cell line U251 and U87 were purchased from ATCC. These cell lines were cultured in Dulbecco's Modified Eagle Medium (Gibco, USA) supplemented with 10\% FBS (Gibco, USA) and 1\% penicillin/streptomycin (Sigma, USA) and maintained at $37^{\circ} \mathrm{C}$ in an atmosphere of $5 \% \mathrm{CO}_{2}$.

\section{Quantitative real-time PCR ( $q R T-P C R)$}

Total RNAs were extracted using Trizol reagent (TaKaRa, Dalian, China) and reversely transcribed into cDNA using a PrimeScript RT Master Mix Perfect Real Time kit (TaKaRa, Dalian, China). PCR was performed using a QuantiTet SYBR Green PCR kit (TaKaRa, Dalian, China) and was measured on an ABI 7900 system (Applied Biosystems, Foster City, CA, USA). The relative expression of specific genes was determined using the equation $2^{-\Delta \Delta \mathrm{Ct}}$. The assay was performed in triplicate for each case.

\section{Cell proliferation assay}

Glioma cells were seeded in 96-well culture plates $(5,000$ cells/well) and cultured for 1,2 , or 3 days. $20 \mu \mathrm{L}$ of MTT (Sigma) was added and the cells were incubated at $37^{\circ} \mathrm{C}$ for 4 hours. Then, the supernatants were removed and $150 \mu \mathrm{L}$ of DMSO was added. Ten minutes after shaking slowly, the optical density (OD) value was determined at $570 \mathrm{~nm}$. 


\section{Colony formation assay}

Glioma cells were seeded in medium mixed with soft agar, and cultured in the incubator for four weeks. Colonies were stained with crystal violet and the number of colonies was calculated.

\section{Apoptosis assay}

Glioma cells were collected, washed twice with PBS and fixed in $80 \%$ ice-cold ethanol in PBS. Then, cells were re-suspended in the staining solution containing FITCconjugated annexin $\mathrm{V}$ antibody and propidium iodide (BD Bioscience). Apoptotic cells were detected on a flow cytometer after incubation for $15 \mathrm{~min}$ at room temperature in the dark.

\section{Transwell invasion assay}

Glioma cells were cultured on the top of Matrigel-coated invasion chambers (BD Bioscience) according to the manufacturer's instruction. After incubating at $37^{\circ} \mathrm{C}$ for $30 \mathrm{~min}$, the cells in the lower surface of the membrane were fixed in methanol and stained with crystal violet. The invasive cells were counted under an inverted microscope.

\section{Western blot}

Glioma cells were collected, lysed in RIRA lysis buffer, and subject to centrifugation at $12,000 \mathrm{rpm}$ for $10 \mathrm{~min}$. Protein lysates were separated by denaturing sodium dodecyl sulfate-polyacrylamide gels and transferred to nitrocellulose membranes (Bio-Rad Laboratories, Hercules, CA). The membranes were probed with the anti-Bcl2 antibody antibodies: (1:5,000, Cell Signaling Technology) and then the secondary antibody labeled by horseradish peroxidase. The protein expression was detected by using the ECL chemiluminescent reagent kit (Amersham GE Healthcare).

\section{Luciferase assay}

The region that contained interacted sites of BCL2 was amplified by PCR from human genomic DNA and inserted into the psiCHECK-2 vector (wild-type) (Promega, Madison, Wisconsin) using the following primers: psiCHECK2-BCL2-F: 5'-CGAGCTCGCTGAATTCT AAATACTGTTAAGGATCTAAGGACAAGCTTG-3'; psiCHECK2-BCL2-R: 5'-CAAGCTTGTCCTTAGAT CCTTAACAGTTTTAGAATTCAGCGAGCTCG-3';
psiCHECK2-BCL2-Mut-F: 5'-CGAGCTCGCTGAAT TCTAAATATGTTTGAGGATCTAAGGACAAGCT TG-3'; psiCHECK2-BCL2-Mut-R: 5'-CAAGCTTGT CCTTAGATCCTATGTGTGTTTAGAATTCAGCG AGCTCG-3'. U87 was plated in 96-well dishes and cotransfected with wild-type or mutant with Lipofectamine 2000. Twenty-four hours later, the cells were lysed and the luciferase activities were measured using dual-luciferase assays (Promega).

\section{Statistical analysis}

Data were expressed as mean $\pm \mathrm{SD}$ and analyzed by the SPSS 17.0 Windows version of software (SPSS). Differences between two groups were analyzed by Student's $t$ test while among multiple groups were analyzed by one-way ANOVA. A value of $\mathrm{P}<0.05$ was considered to be statistically significant.

\section{Results}

miR-30c-5p suppresses glioma cell proliferation

Firstly, we explored the expression pattern of miR-30c$5 \mathrm{p}$ in 20 paired clinical glioma samples and the adjacent normal tissues. Results showed that the relative expression of miR-30c-5p was significantly decreased in glioma tissues compared with normal tissues (Figure 1A). To explore the biological role of miR-30c-5p in glioma behaviors, we evaluated the effect of miR-30c-5p on tumor growth in glioma cells overexpressing or downregulating miR-30c-5p (Figure 1B). Consequently, MTT assay revealed that upregulation of miR-30c-5p significantly inhibited cell viability; whereas knockdown of miR-30c-5p promoted cell viability, in U87 and U251 glioma cells in a time-dependent manner (Figure 1C,D). Furthermore, ectopic expression of miR-30c-5p dramatically reduced colony formation ability of glioma U87 (Figure 2A,B) and U251 cells (Figure 2A,C). Conversely, transfection with miR-30c-5p inhibitors into glioma cells led to elevated colony formation abilities in U87 (Figure $2 A, B$ ) and U251 cells (Figure 2A,C). Collectively, these data reveal that miR-30c-5p serves as an inhibitor on glioma cell proliferation.

\section{miR-30c-5p induces glioma cell apoptosis}

It is well established that cell apoptosis is closely associated with proliferation ability. Thus, we further examined the 
A

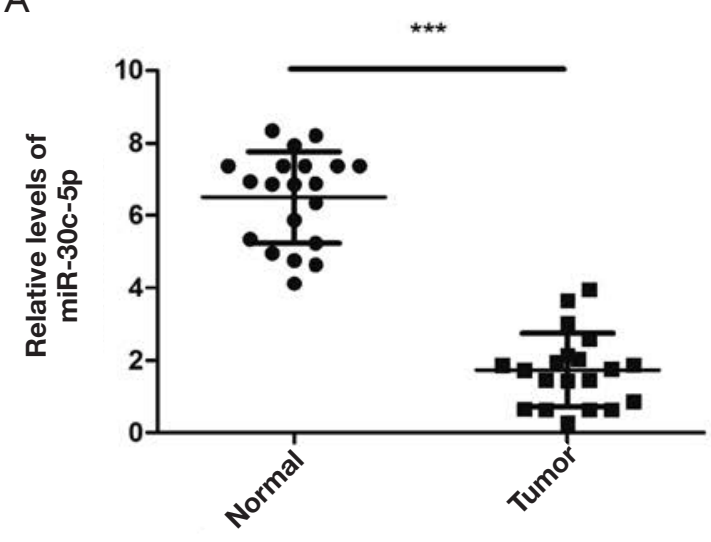

B

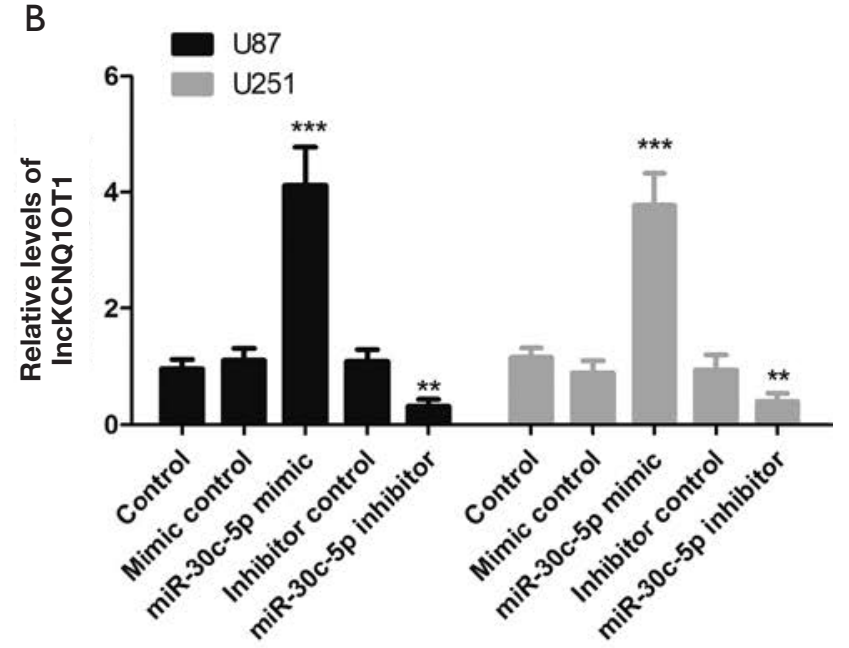

C
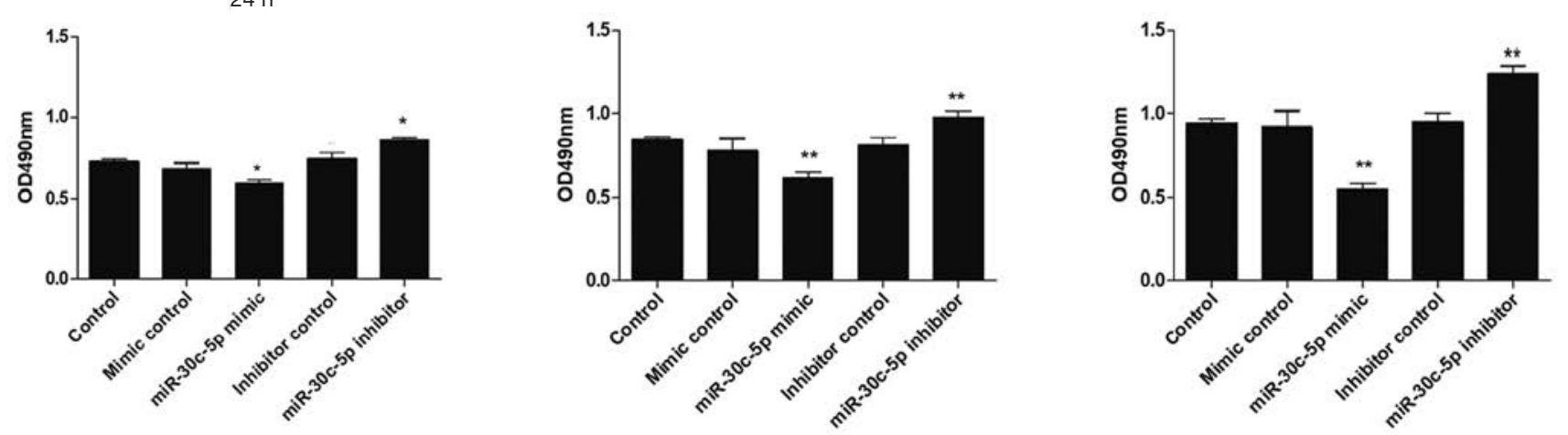

D

$24 \mathrm{~h}$

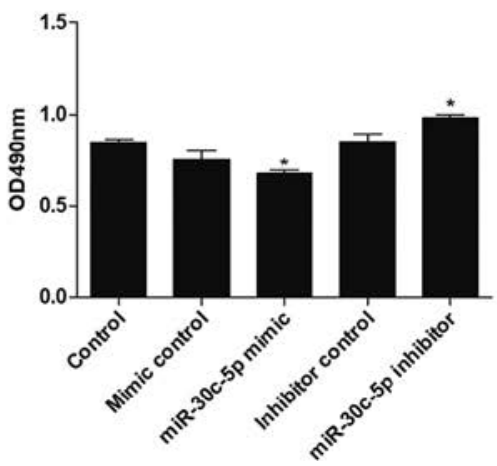

$48 \mathrm{~h}$

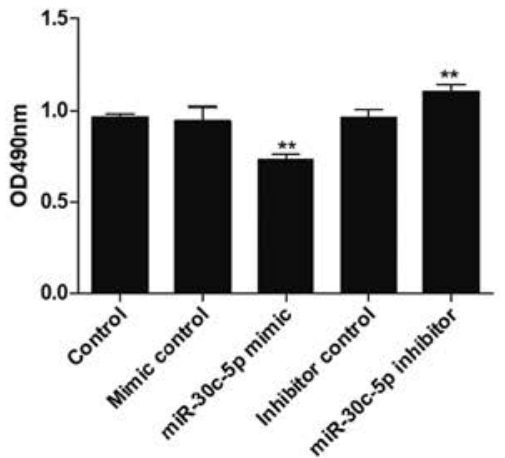

$72 \mathrm{~h}$

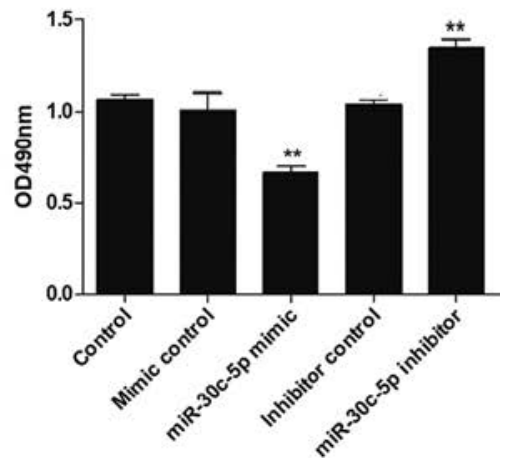

Figure 1 miR-30c-5p suppresses the cell viability of U87 and U251 glioma cells. (A) Expression of miR-30c-5p in glioma tissues and normal tissues was determined using qRT-PCR. ***, $\mathrm{P}<0.001$, compared to normal. Two glioma cell lines including U87 and U251 were transfected with miR-30c-5p mimic or inhibitors (B). The cell viability of glioma cells was evaluated by MTT assay in different time points (C,D). *, $\mathrm{P}<0.05 ;{ }^{* *}, \mathrm{P}<0.01$. 
A
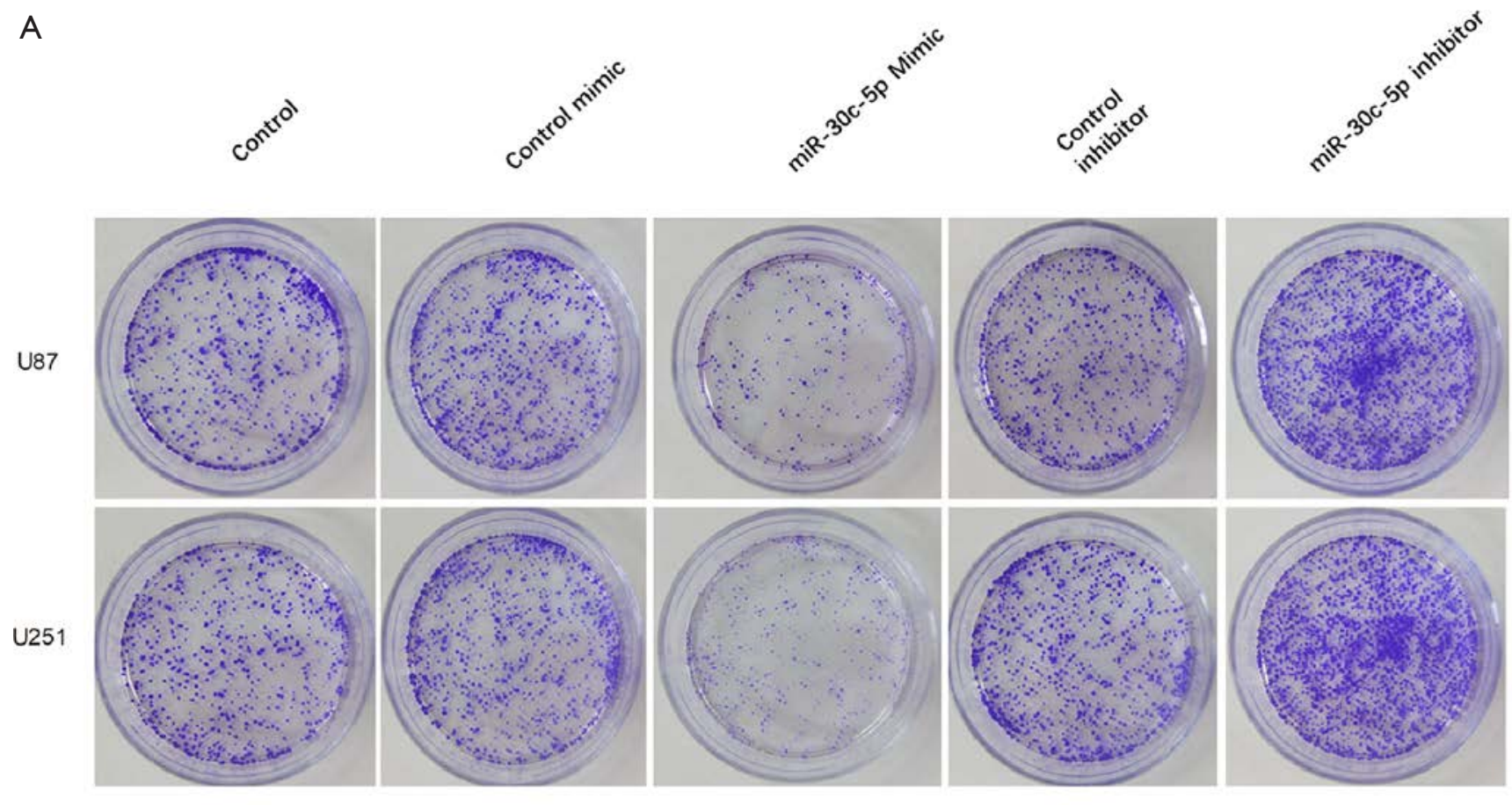

B

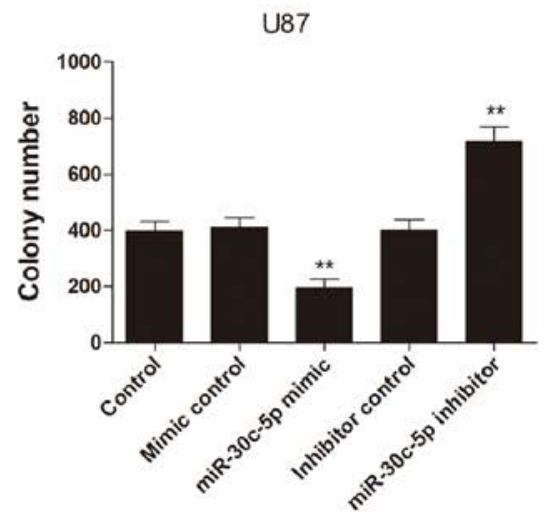

C

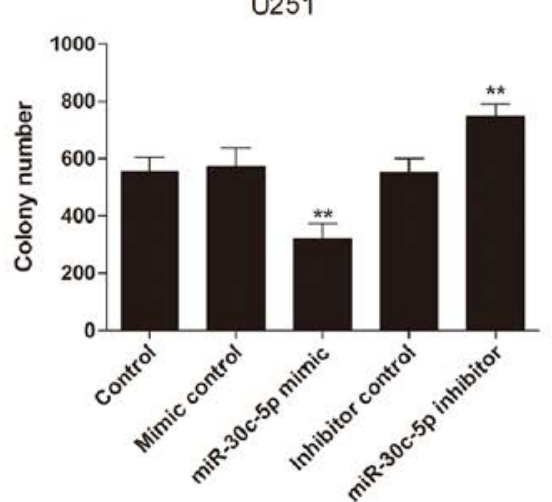

Figure 2 miR-30c-5p suppresses colony formation of U87 and U251 glioma cells. Glioma cell lines U87 (A,B) and U251 (A,C) were transfected with miR-30c-5p mimic or inhibitors. The colony formation of glioma cells was evaluated by colony formation assay. Colonies were stained with crystal violet. Magnification $5 \times{ }^{* *}, \mathrm{P}<0.01$.

effect of miR-30c-5p on glioma cell apoptosis. Cytometry analysis showed that ectopic expression of miR-30c$5 \mathrm{p}$ obviously increased the numbers of apoptosis in U87 (Figure $3 A, B$ ) and $\mathrm{U} 251$ cells (Figure $3 A, C$ ) compared to negative controls. Conversely, inhibition of miR-30c$5 \mathrm{p}$ decreased the apoptotic rate of glioma cells in U87 (Figure $3 A, B$ ) and $\mathrm{U} 251$ cells (Figure $3 A, C$ ). These results showed that miR-30c-5p inhibits proliferation of glioma cells through induction of apoptosis.

\section{miR-30c-5p inhibits glioma cell invasive potential}

To determine if miR-30c-5p affected the invasiveness of glioma cells, transwell assay was performed after transfection with miR-30c-5p mimic or inhibitor into the U87 and U251 cells. As a result, miR-30c-5p mimic- 
A
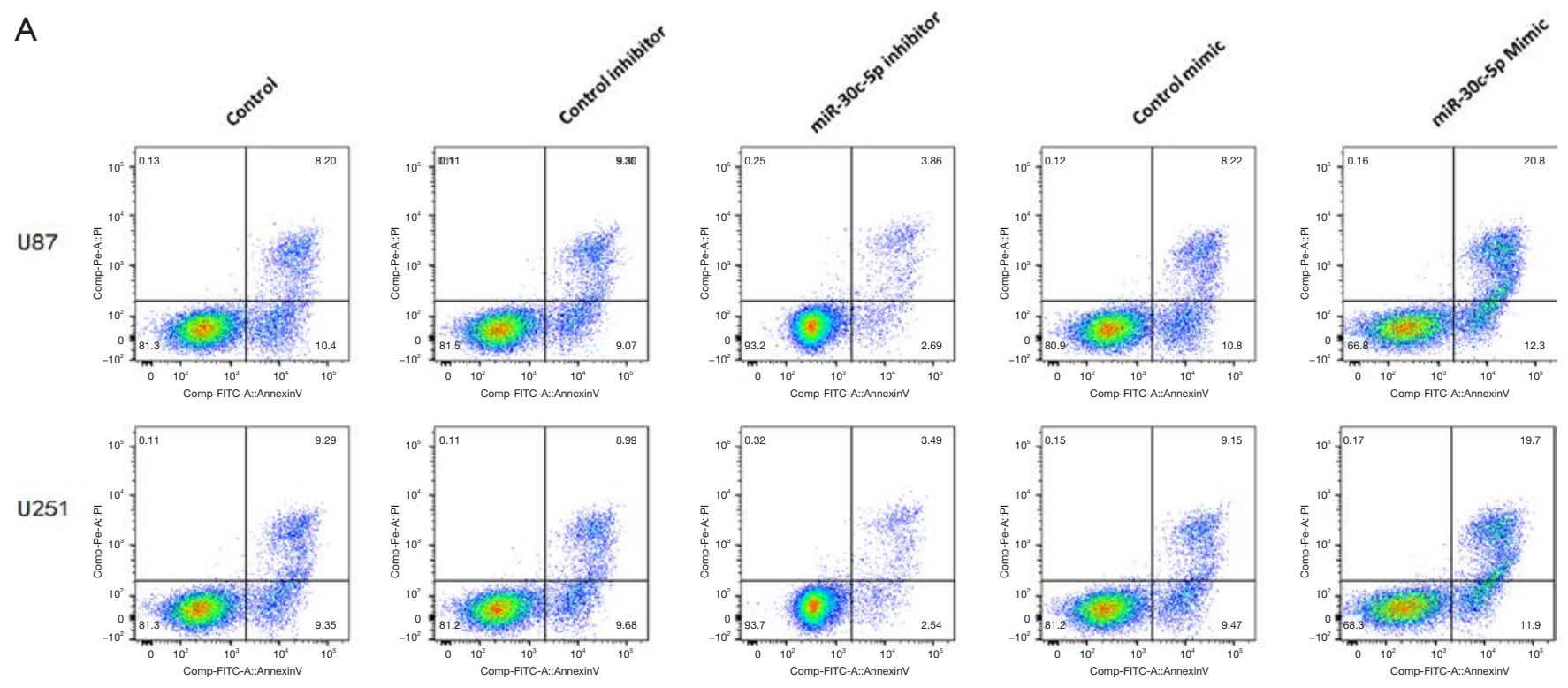

B

C
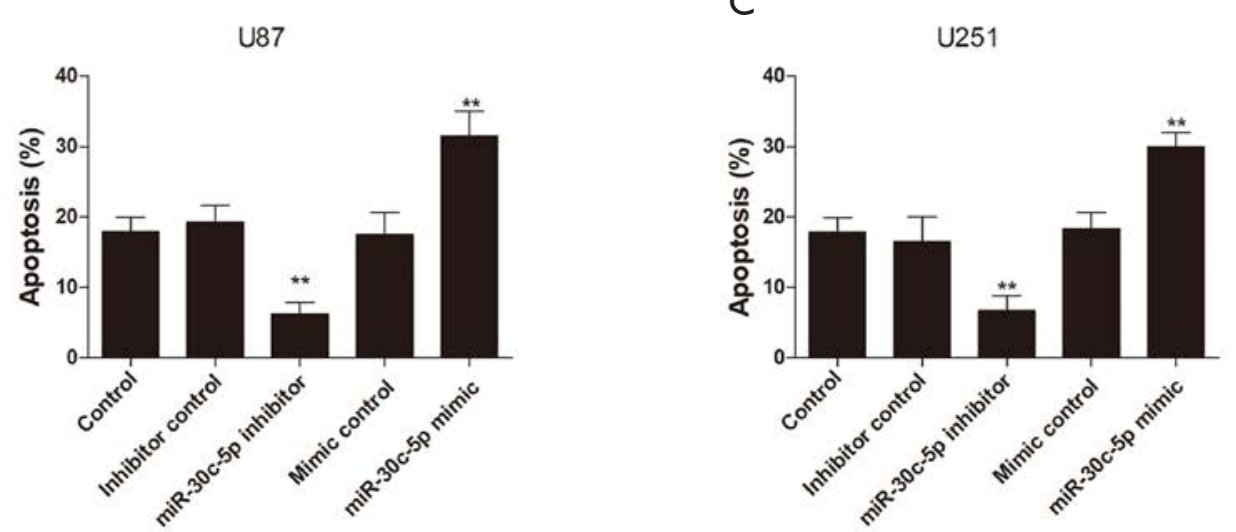

Figure 3 miR-30c-5p induces glioma cell apoptosis of U87 and U251 glioma cells. Glioma cell lines U87 and U251 were transfected with miR-30c-5p mimic or inhibitors. Flow cytometry was used to detect the cell apoptosis of U87 (A,B) and U251 (A,C) glioma cells. **, $\mathrm{P}<0.01$.

transfected cells exhibited fewer invasive cells; meanwhile, miR-30c-5p inhibitor-transfected cells presented with enhanced invasive ability in U87 (Figure $4 A, B$ ) and U251 (Figure $4 A, C$ ) cells. Collectively, these data suggest that miR-30c-5p strongly suppresses the invasion of glioma cells.

\section{Bcl2 is a direct target of miR-30c-5p}

Bioinformatics software was used to identify the potential target genes of miR-30c-5p. Consequently, the 3'UTR of
Bcl2 gene was found to contain the conserved regions that may serve as binding sites for miR-30c-5p (Figure 5A). In addition, we found that miR-30c-5p overexpression significantly decreased the expression of $\mathrm{Bcl} 2$, and transfection with miR-30c-5p inhibitor increased the protein levels of Bcl2 in U87 and U251 cells (Figure 5B). To further verify that $\mathrm{Bcl} 2$ is a potential target of miR$30 c-5 p$, we generated luciferase reporters that contained the 3'UTR or a mutated sequence within the biding site of the $\mathrm{Bcl} 2$ gene. As a result, the reporter activity of wildtype Bcl2-3'UTR was reduced but the luciferase activity of 
A

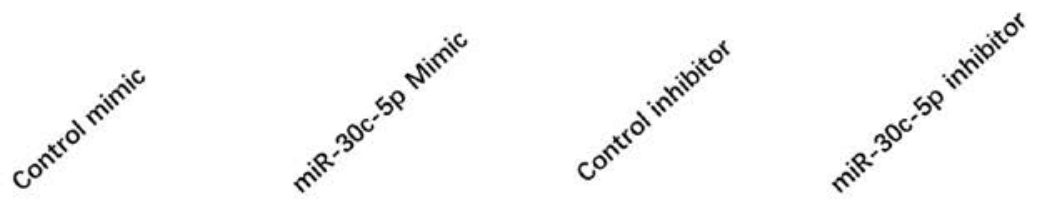

U87

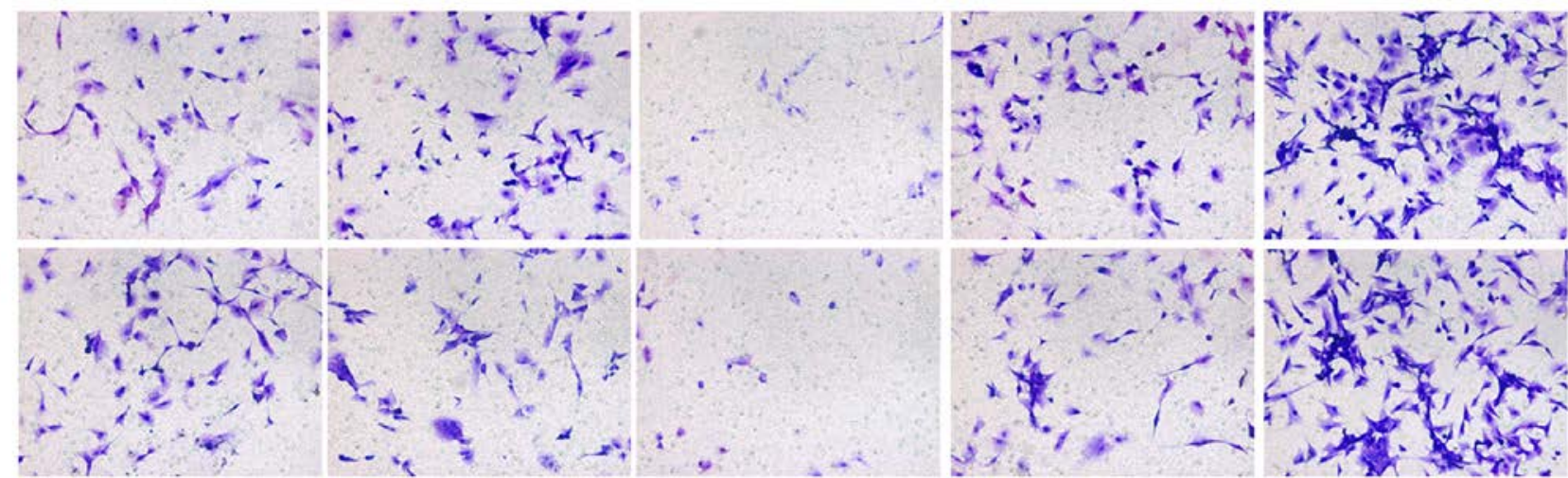

B

U87

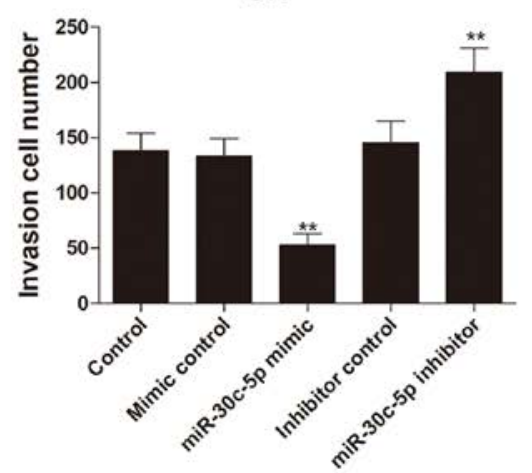

C

U251

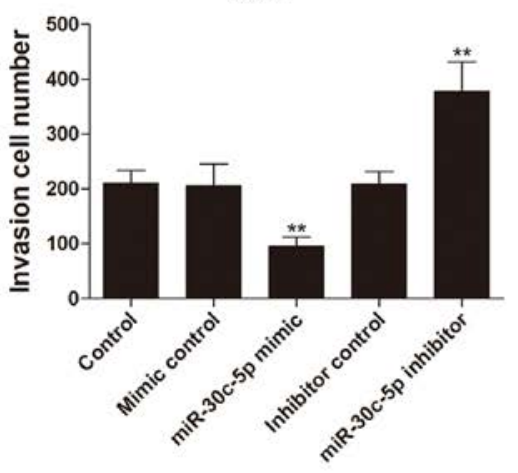

Figure 4 miR-30c-5p inhibits glioma cell invasive potential of U87 and U251 glioma cells. Glioma cell lines U87 and U251 were transfected with miR-30c-5p mimic or inhibitors. Transwell invasion assay was used to evaluate the invasive potential of U87 (A,B) and U251 (A,C) glioma cells. **, $\mathrm{P}<0.01$. Magnification $200 \times$.

mutated Bcl2-3'UTR remained unchanged in miR-30c-5p mimic-transfected cells (Figure 5C). Given that Bcl2 and caspase pathways are involved in apoptosis, we evaluated the expression of caspase-3/8/9 in glioma cells. Data showed that overexpression of miR-30c-5p in U87 cells dramatically led to caspase 3/9 activation, as evidenced by elevated cleaved caspase 3/9; whereas knockdown of miR$30 \mathrm{c}-5 \mathrm{p}$ decreased levels of cleaved caspase 3/9 (Figure 5D). However, the expression of caspase 3, 8 and 9 remained unchanged in U87 cells treated with miR-30c-5p inhibitor or mimic. Collectively, these findings confirm that miR-
$30 c-5 p$ targets Bcl2 and activates caspase pathway in glioma cells.

\section{miR-30c-5p regulates glioma cellular behaviors via Bcl2}

Furthermore, we explored whether miR-30c-5p regulates glioma cellular behaviors via targeting Bcl2. U87 and U251 cells were co-transfected with miR-30c-5p mimic or inhibitor alone, or miR-30c-5p inhibitor plus Bcl-2 overexpression and miR-30c-5p mimics plus Bcl-2 knockdown. Consequently, we found that miR-30c-5p 

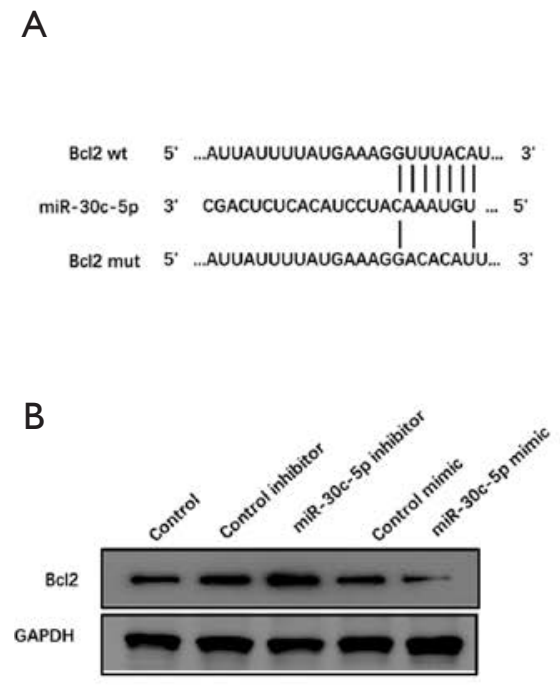
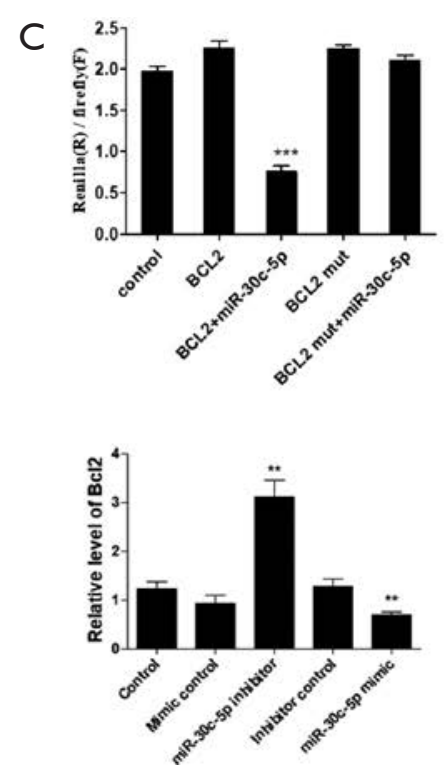

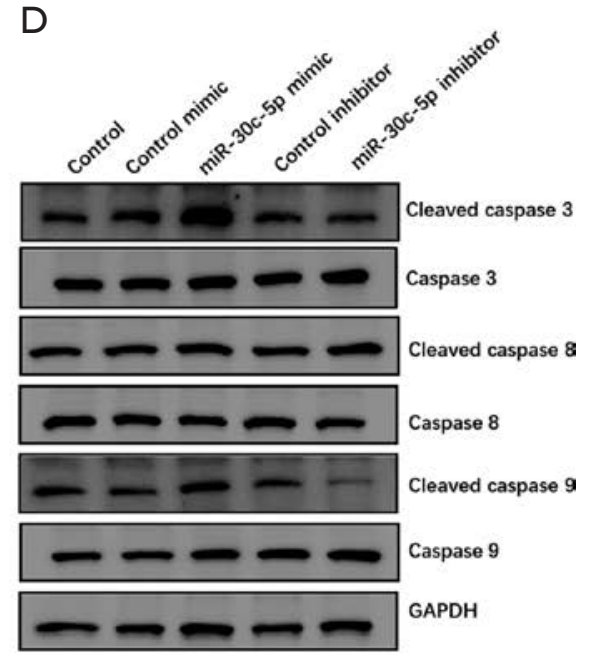

Figure $5 \mathrm{Bcl} 2$ is a direct target of miR-30c-5p in glioma cells. The 3 'UTR of Bcl2 gene contained the binding sites for miR-30c-5p (A). U87 cells were transfected with miR-30c-5p mimic or inhibitors. (B) The protein expression of Bcl 2 was detected by western blot and then quantified to the control GAPDH. (C) Luciferase reporter assay was used to validate that Bcl2 is a direct target of miR-30c-5p. (D) The protein expression of caspase 3/8/9 and cleaved caspase 3/8/9 was detected by western blot. ${ }^{* *}, \mathrm{P}<0.01 ;{ }^{* * *}, \mathrm{P}<0.001$.

reduced the colony formation ability, and knockdown of Bcl2 combined with miR-30c-5p mimics further suppressed colony formation of U87 and U251 glioma cells; miR$30 c-5 p$ inhibitors increased colony formation ability, and restored $\mathrm{Bcl} 2$ expression further enhanced glioma cell growth (Figure 6A,B). Consistently, miR-30c-5p inhibited migration, and knockdown of Bcl2 further suppressed migration of glioma cells; by contrast, miR$30 \mathrm{c}-5 \mathrm{p}$ inhibitors increased migration, and restored $\mathrm{Bcl} 2$ expression further enhanced migration (Figure $6 C, D$ ). As shown in Figure 7A,B,C,D, overexpression of miR-30c-5p induced apoptosis and knockdown of Bcl2 further increased the number of apoptotic cells; by contrast, miR-30c-5p inhibitors decreased apoptosis and restored $\mathrm{Bcl} 2$ expression further suppressed glioma cell apoptosis in U87 and U251 glioma cells. Collectively, these findings validate that miR$30 \mathrm{c}-5 \mathrm{p}$ regulates cellular behaviors of glioma cells by targeting Bcl2.

\section{Discussion}

In the present study, we demonstrated that glioma cell growth and invasion were inhibited, and apoptosis was induced by overexpression of miR-30c-5p. These observations elicited a further study for the mechanisms of miR-30c-5p responsible for these functions.

It is well established that miRNAs post-transcriptionally regulate the downstream targets to interfere with cellular behaviors, such as proliferation, apoptosis, migration, and drug resistance $(16,17)$. By this means, miRNAs play an important role in tumor initiation and progression. For instance, miR-2 1 is found to be elevated in colorectal cancers, and enforced expression of miR-21 promoted tumor cell migration and metastasis via targeting PDCD4 (18). Thus, miR-21 acts as an oncogene to exhibit regulatory effects in cancer development under this context. On the other hand, miRNAs also function as tumor suppressing factors, and are constantly expressed in a lower level. For example, let-7, a tumor suppressor gene, is absent in lung cancers and could inhibit cancer progression by inhibiting downstream target k-Ras and c-MYC (19).

miR-30c-5p is a member of the miR-30c family and has been reported to be essential for cellular behaviors. A recent study suggests that miR-30c-5p directly targets FASN and ameliorates hepatic steatosis in leptin receptor-deficient (db/db) mice (20). In addition, miRNA-30c-5p inhibits NLRP3 inflammasome-mediated endothelial cell pyroptosis by downregulating FOXO3 in ox-LDL-treated human 
A

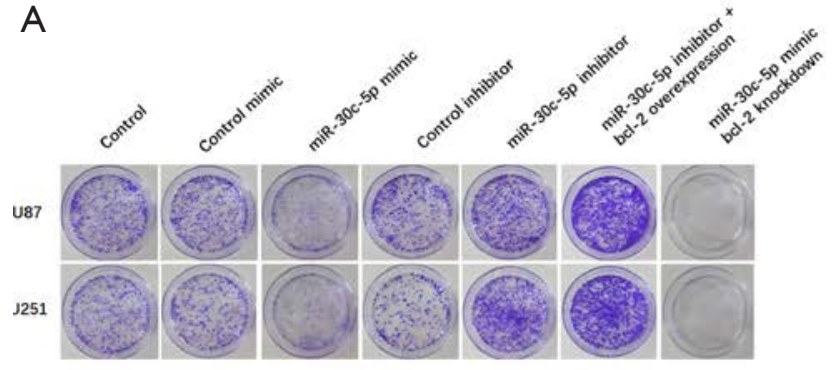

B

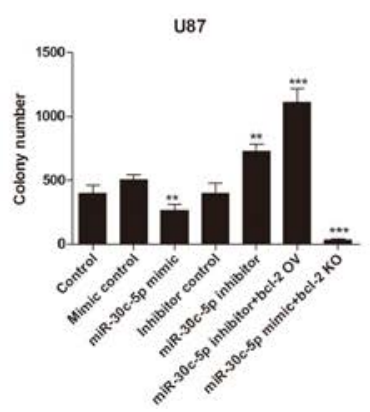

$\mathrm{U} 251$

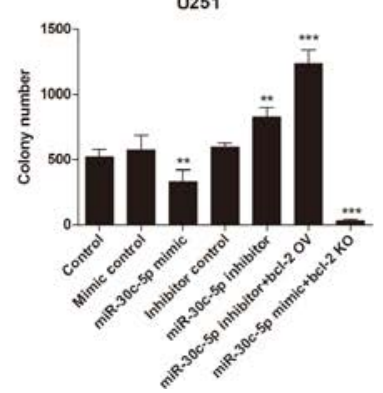

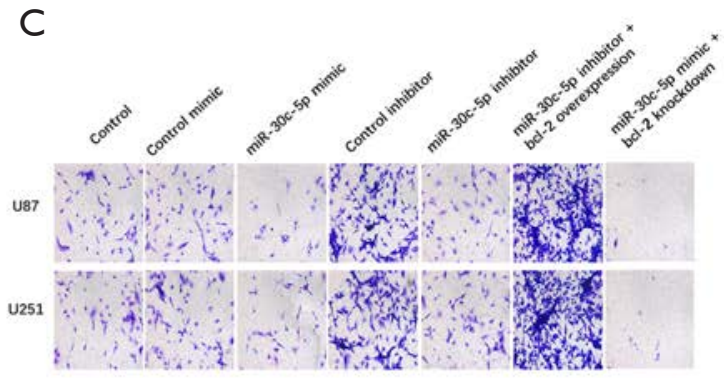

D

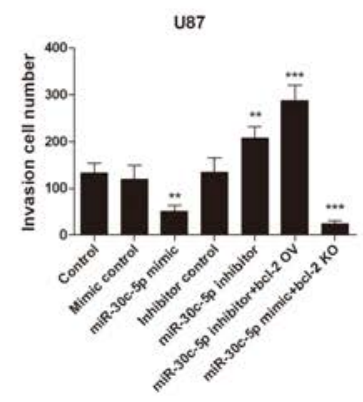

U251

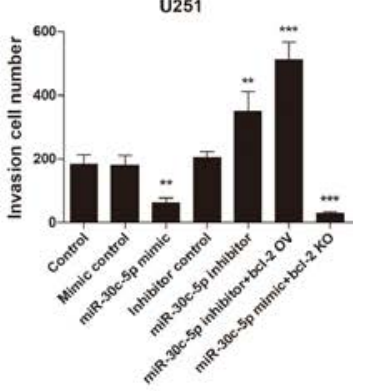

Figure 6 miR-30c-5p regulates glioma cell colony formation and invasion via Bcl2. Glioma cell lines U87 and U251 were transfected with miR-30c-5p mimic or inhibitor alone, or miR-30c-5p inhibitor plus Bcl-2 overexpression and miR-30c-5p mimics plus Bcl-2 knockdown. Cell colony formation and migration were detected by colony formation assay (A,B) and Transwell invasion assay (C,D), respectively. Colonies and invaded cells were stained with crystal violet. Magnification $200 \times$. ${ }^{* *}, \mathrm{P}<0.01 ;{ }^{* *}, \mathrm{P}<0.001$.

aortic endothelial cells, suggesting a potential therapeutic value in atherosclerosis (21). Moreover, miRNA-30c-5p has been reported to be down-regulated in breast, lung, gastric and ovarian cancers (22), indicating its potential tumor suppression effect in human cancers. Indeed, down-regulation of miR-30c-5p is associated with clinical metastasis and poor prognosis in gastric cancer patient. Mechanical investigation revealed miR-30c-5p could inhibit migration and epithelial to mesenchymal transition (EMT) process via targeting metastasis-associated protein 1 (MTA1) (15). In ovarian cancer cells, miR-30c-5p is shown to suppress cisplatin resistance and EMT through targeting DNA methyltransferase-1 (DNMT1) expression (23). Previous studies reported that miRNA-30c could be involved in self-renewal and neural differentiation in glioma cells (16). However, there are still no evidence of miR$30 c-5 p$ in the pathogenesis and progression of glioma. In the present study, we found that enforced expression of miR-30c-5p significantly suppressed the proliferation and colony formation, and induced cell apoptosis in glioma cells. In contrast, inhibition of miR-30c-5p promoted cell growth and inhibited apoptosis in tumor cells. Regarding mechanical investigation, it is well known that apoptosis can be initiated by the extrinsic pathway, which is mediated by activation of caspase 3, 8 and 9 (12-14). Our data showed that miR-30c-5p promoted the activation of caspase 3 and caspase 9, but not caspase 8 in glioma cells. Furthermore, it has been reported that miR-30c-5p is associated with tumor cell invasion and distant metastasis (15). Consistently, our study observed that miR-30c-5p strongly suppresses the invasion of glioma cells, representing its potential role in metastatic glioma treatment.

Apoptosis is triggered by $\mathrm{Bcl}-2$ superfamily members, including Bax, Bcl-2 and Bcl-xL (24). It has been demonstrated that expression of Bcl-2 may be targeted by several miRNAs in numerous types of tumor cells, including human hepatocellular carcinoma cells, pancreatic cancer cells, and gastric cancer cells (25). In the present study, the putative binding site of miR-30c-5p in Bcl-2 3'UTR was identified by bioinformatic analysis. Western blot showed that the protein expression of Bcl-2 was significantly decreased following treatment with miR- 
A
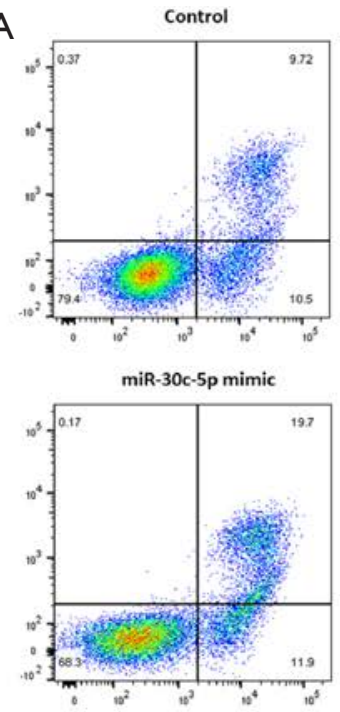

B
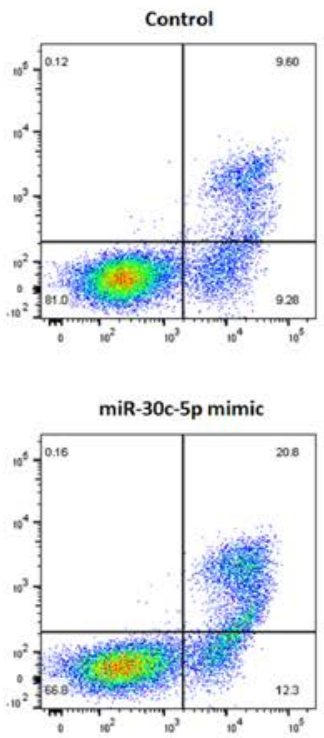

C

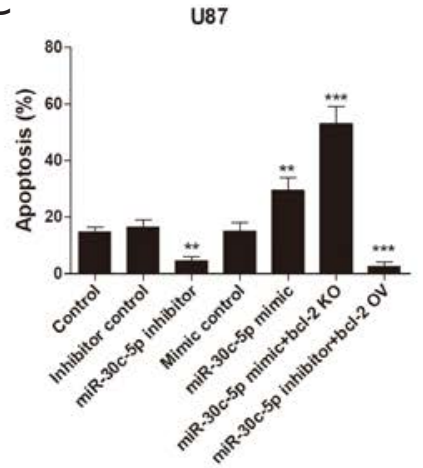

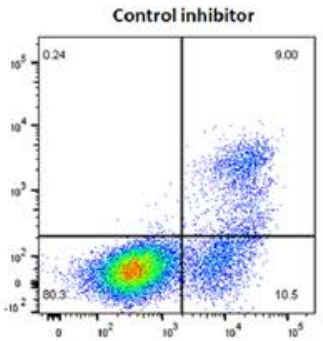

miR-30c-5p mimic + bcl2 knockdown
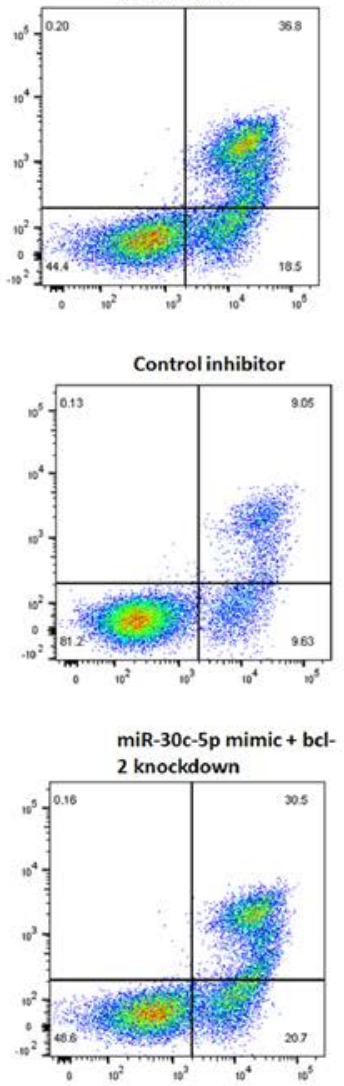

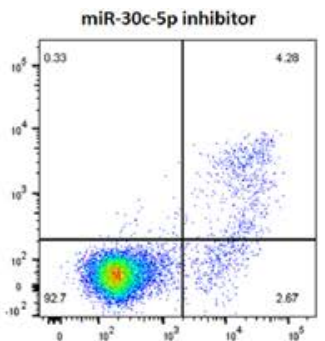

miR-30c-5p inhibitor bcl-2 overexpression
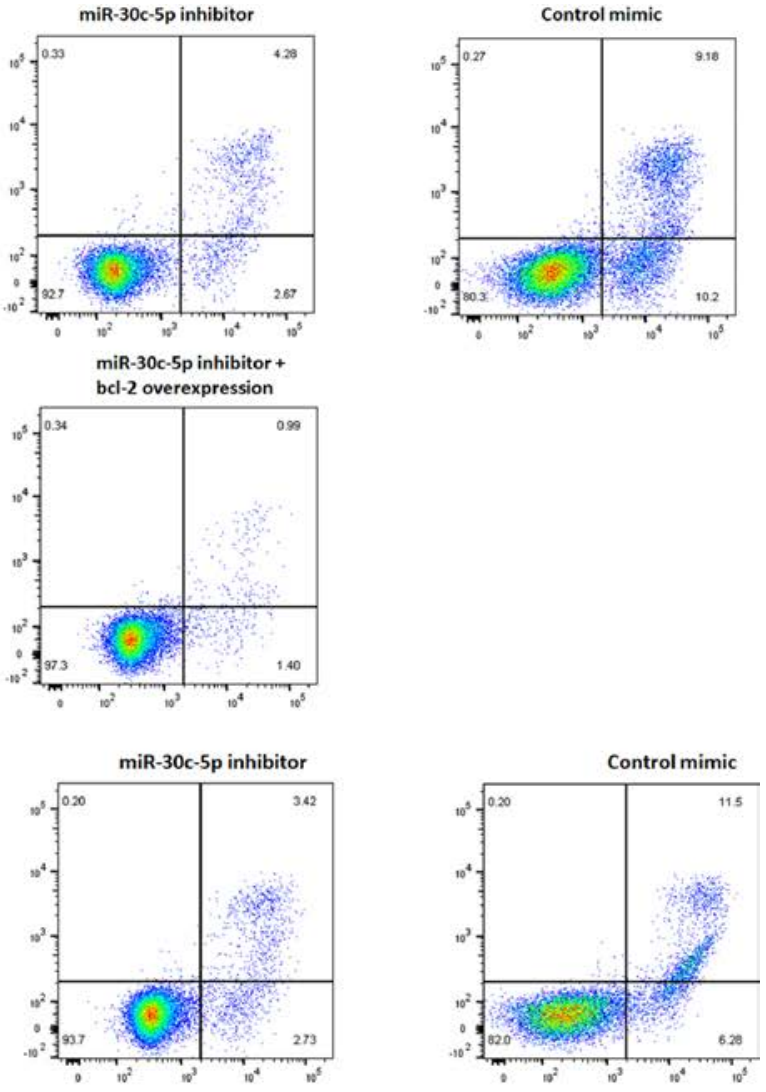

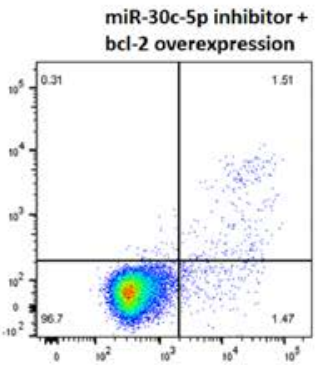

D U251

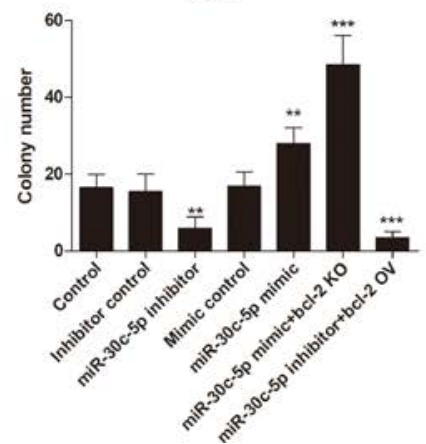

Figure 7 miR-30c-5p induces glioma cell apoptosis of glioma cells via Bcl2. Glioma cell lines U87 and U251 were transfected with miR$30 \mathrm{c}-5 \mathrm{p}$ mimic or inhibitor alone, or miR-30c-5p inhibitor plus Bcl-2 overexpression and miR-30c-5p mimics plus Bcl-2 knockdown. Flow cytometry was used to detect the cell apoptosis of $\mathrm{U} 87(\mathrm{~A}, \mathrm{C})$ and $\mathrm{U} 251$ (B,D) glioma cells. ${ }^{* *}, \mathrm{P}<0.01$; ${ }^{* * *}, \mathrm{P}<0.001$. 
$30 c-5 p$ mimics and increased after miR-30c-5p inhibitor treatment. Moreover, luciferase reporter assays indicated that transfection of miR-30c-5p led to a marked reduction of luciferase activity, but had no effect on Bcl-2 3'-UTR mutated fragment. Furthermore, we found that miR-30c$5 p$ promoted apoptosis and inhibited colony formation and migration, and knockdown of Bcl2 further increased the number of apoptotic cells and suppressed colony formation and migration of glioma cells; by contrast, miR$30 c-5 p$ inhibitors decreased apoptosis and increased colony formation and migration, and restored $\mathrm{Bcl} 2$ expression further suppressed glioma cell apoptosis and enhanced colony formation and migration. Collectively, these results suggested that miR-30c-5p regulates cellular behaviors by targeting Bcl-2 in glioma cells.

In summary, these findings provide new insights into the role of miR-30c-5p in human gliomas. Upregulation of miR-30c-5p could suppress glioma growth and invasion by targeting Bcl2, suggesting that miR-30c-5p might represent a novel target for glioma therapy.

\section{Acknowledgments}

Funding: This study was supported by Suzhou Science and Technology Project (No. SYS201630).

\section{Footnote}

Reporting Checklist: The authors have completed the MDAR reporting checklist. Available at http://dx.doi.org/10.21037/ tcr-19-2957

Data Sharing Statement: Available at http://dx.doi. org/10.21037/tcr-19-2957

Conflicts of Interest: All authors have completed the ICMJE uniform disclosure form (available at http://dx.doi. org/10.21037/tcr-19-2957). The authors have no conflicts of interest to declare.

Ethical Statement: The authors are accountable for all aspects of the work in ensuring that questions related to the accuracy or integrity of any part of the work are appropriately investigated and resolved. The study was conducted in accordance with the Declaration of Helsinki (as revised in 2013). This study was approved by the Ethics Committee of the Second Affiliated Hospital of Soochow University [No. JD-LK-2018-027-02], and all participants provided written informed consent.

Open Access Statement: This is an Open Access article distributed in accordance with the Creative Commons Attribution-NonCommercial-NoDerivs 4.0 International License (CC BY-NC-ND 4.0), which permits the noncommercial replication and distribution of the article with the strict proviso that no changes or edits are made and the original work is properly cited (including links to both the formal publication through the relevant DOI and the license). See: https://creativecommons.org/licenses/by-nc-nd/4.0/.

\section{References}

1. Omuro A, DeAngelis LM. Glioblastoma and other malignant gliomas: a clinical review. JAMA 2013;310:1842-50.

2. Touat M, Idbaih A, Sanson M, et al. Glioblastoma targeted therapy: updated approaches from recent biological insights. Ann Oncol 2017;28:1457-72.

3. Furnari FB, Fenton T, Bachoo RM, et al. Malignant astrocytic glioma: genetics, biology, and paths to treatment. Genes Dev 2007;21:2683-710.

4. Mehta A, Baltimore D. MicroRNAs as regulatory elements in immune system logic. Nat Rev Immunol 2016;16:279-94.

5. Harrandah AM, Mora RA, Chan EKL. Emerging microRNAs in cancer diagnosis, progression, and immune surveillance. Cancer Lett 2018;438:126-32.

6. Tang J, Li Y, Wang J, et al. Molecular mechanisms of microRNAs in regulating epithelial-mesenchymal transitions in human cancers. Cancer Lett 2016;371:301-13.

7. Ghai V, Wang K. Recent progress toward the use of circulating microRNAs as clinical biomarkers. Arch Toxicol 2016;90:2959-78.

8. Wu XQ, Dai Y, Yang Y, et al. Emerging role of microRNAs in regulating macrophage activation and polarization in immune response and inflammation. Immunology 2016;148:237-48.

9. Wu W, Zhang X, Liao Y, et al. miR-30c negatively regulates the migration and invasion by targeting the immediate early response protein 2 in SMMC-7721 and HepG2 cells. Am J Cancer Res 2015;5:1435-46.

10. Zheng Z, Guan M, Jia Y, et al. The coordinated roles of miR-26a and miR-30c in regulating TGFbeta1induced epithelial-to-mesenchymal transition in diabetic nephropathy. Sci Rep 2016;6:37492. 
11. Chen X, Chen RX, Wei WS, et al. PRMT5 Circular RNA Promotes Metastasis of Urothelial Carcinoma of the Bladder through Sponging miR-30c to Induce Epithelial-Mesenchymal Transition. Clin Cancer Res 2018;24:6319-30.

12. Liu S, Li X, Zhuang S. MiR-30c impedes glioblastoma cell proliferation and migration by targeting SOX9. Oncol Res 2019;27:165-71.

13. Lai YH, Chen J, Wang XP, et al. Collagen triple helix repeat containing-1 negatively regulated by microRNA30c promotes cell proliferation and metastasis and indicates poor prognosis in breast cancer. J Exp Clin Cancer Res 2017;36:92.

14. Zhao Y, Yin Z, Li H, et al. MiR-30c protects diabetic nephropathy by suppressing epithelial-to-mesenchymal transition in db/db mice. Aging Cell 2017;16:387-400.

15. Cao JM, Li GZ, Han M, et al. MiR-30c-5p suppresses migration, invasion and epithelial to mesenchymal transition of gastric cancer via targeting MTA1. Biomed Pharmacother 2017;93:554-60.

16. Quintavalle C, Donnarumma E, Iaboni M, et al. Effect of $\mathrm{miR}-21$ and $\mathrm{miR}-30 \mathrm{~b} / \mathrm{c}$ on TRAIL-induced apoptosis in glioma cells. Oncogene 2013;32:4001-8.

17. Engels BM, Hutvagner G. Principles and effects of microRNA-mediated post-transcriptional gene regulation. Oncogene 2006;25:6163-9.

18. Ferraro A, Kontos CK, Boni T, et al. Epigenetic regulation of in colorectal cancer: ITGB4 as a novel miR-21 target and a three-gene network (miR-21-ITGBeta4-PDCD4) as predictor of metastatic tumor potential. Epigenetics 2014;9:129-41.

19. Kolenda T, Przybyła W, Teresiak A, et al. The mystery of let-7d - a small RNA with great power. Contemp Oncol (Pozn) 2014;18:293-301.

20. Fan J, Li H, Nie X, et al. MiR-30c-5p ameliorates hepatic steatosis in leptin receptor-deficient $(\mathrm{db} / \mathrm{db})$ mice via down-regulating FASN. Oncotarget 2017;8:13450-63.

21. Li P, Zhong X, Li J, et al. MicroRNA-30c-5p inhibits NLRP3 inflammasome-mediated endothelial cell pyroptosis through FOXO3 down-regulation in atherosclerosis. Biochem Biophys Res Commun 2018;503:2833-40.

22. Irani S, Hussain MM. Role of microRNA-30c in lipid metabolism, adipogenesis, cardiac remodeling and cancer. Curr Opin Lipidol 2015;26:139-46.

23. Han X, Zhen S, Ye Z, et al. A Feedback Loop Between miR-30a/c-5p and DNMT1 Mediates Cisplatin Resistance in Ovarian Cancer Cells. Cell Physiol Biochem 2017;41:973-86.

24. Janumyan YM, Sansam CG, Chattopadhyay A, et al. Bcl$\mathrm{xL} / \mathrm{Bcl}-2$ coordinately regulates apoptosis, cell cycle arrest and cell cycle entry. EMBO J 2003;22:5459-70.

25. Delbridge AR, Grabow S, Strasser A, et al. Thirty years of BCL-2: translating cell death discoveries into novel cancer therapies. Nat Rev Cancer 2016;16:99-109.
Cite this article as: Yuan LQ, Zhang T, Xu L, Han H, Liu SH. miR-30c-5p inhibits glioma proliferation and invasion via targeting Bcl2. Transl Cancer Res 2021;10(1):337-348. doi: $10.21037 /$ tcr-19-2957 